\title{
Assistência de enfermagem à paciente com proposta cirúrgica de hemicorporectomia: relato de caso
}

Nursing care to patients with a surgical proposal of hemicorporectomy: case report

Atención de enfermería para los pacientes con una propuesta quirúrgica para hemicorporectomía: informe de caso

Jamil Michel Miranda do Vale ${ }^{1 *}$, Clarissa Porfírio Mendes², Genize do Socorro Teixeira da Silva ${ }^{2}$, Mary Elizabeth de Santana ${ }^{1,3}$.

\section{RESUMO}

Objetivo: Descrever a assistência de enfermagem um paciente com proposta cirúrgica de hemicorporectomia. Detalhamentos de Caso: Relato de caso, realizado em julho de 2019, em um hospital de referência no tratamento de câncer. Utilizou-se para coleta de dados um roteiro estruturado nos aspectos do cuidado. A análise dos dados foi subsidiada pelo Processo de Enfermagem e demais bases conceituais que debatem a temática. O planejamento da assistência foi realizado levando em consideração o fator biológico, mas também necessidade humanas adentrando no campo das interações, reflexões e conhecimento no contexto social, cultural e espiritual. Considerações finais: A taxonomia destacou-se como ferramenta de utilização para direcionamento das intervenções de enfermagem na promoção da saúde do usuário considerando os fatores sociais, culturais e espirituais, obtendo-se resultados positivos, quanto ao alcance dos resultados esperados com melhora significativa no enfrentamento da doença e continuidade no tratamento.

Palavras-chave: Hemicorporectomia, Carcinoma de células escamosas, Neoplasias pélvicas, Enfermagem oncológica, Procedimentos cirúrgicos operatórios.

\begin{abstract}
Objective: To describe the nursing care for a patient with surgical proposal of hemicorporectomy. Case Detail: case report, conducted in July 2019, at a hospital reference in cancer treatment. A structured script was used for data collection. Data analysis will be supported by the Nursing Process and other conceptual bases that debate the theme. The planning of care was carried out taking into consideration the biological factor, but also human need entering the field of interactions, reflections and knowledge in the social, cultural and spiritual context. Final Considerations: The taxonomy stood out as a tool of use for directing nursing interventions in the promotion of user health considering the social, cultural and spiritual factors, obtaining positive results, as to the expected results with significant improvement in coping with the disease and continuity of treatment.
\end{abstract}

Keywords: Hemicorporectomy, Carcinoma squamous cell, Pelvic neoplasms, Oncology nursing, Surgical procedures operative.

\section{RESUMEN}

Objetivo: Describir la atención de enfermería para un paciente con una propuesta quirúrgica para hemicorporectomía. Detalle del caso: Informe del caso, realizado en julio de 2019, en un hospital de referencia en el tratamiento del cáncer. Se utilizó un script estructurado para la recopilación de datos. El análisis de datos

\footnotetext{
${ }^{1}$ Universidade Federal do Pará (UFPA), Belém-PA. *E-mal: jamilvale@yahoo.com

${ }^{2}$ Hospital Ophir Loyola, Belém-PA.

3 Universidade do Estado do Pará (UEPA), Belém-PA.
} 
será subsidiado por el Proceso de Enfermería y otras bases conceptuales que debaten el tema. La planificación de la atención se llevó a cabo teniendo en cuenta el factor biológico, pero también la necesidad humana de ingresar al campo de las interacciones, reflexiones y conocimientos en el contexto social, cultural y espiritual. Consideraciones finales: La taxonomía se destacó como una herramienta de uso para dirigir las intervenciones de enfermería en la promoción de la salud del usuario considerando los factores sociales, culturales y espirituales, obteniendo resultados positivos, en cuanto a los resultados esperados con una mejora significativa en el manejo de la enfermedad. continuidad en el tratamiento.

Palabras clave: Hemicorporectomía, Carcinoma de células escamosas, Neoplasias pélvicas, Enfermería oncológica, Procedimientos quirúrgicos operativos.

\section{INTRODUÇÃO}

As doenças crônicas não transmissíveis (DCNTs) são agora responsáveis pela maioria das mortes globais, e espera-se que o câncer seja a principal causa de morte e a única barreira mais importante para aumentar a expectativa de vida. O câncer apresenta-se como a primeira ou segunda causa de morte em 91 dos 172 países, no Brasil, estima-se 600 mil casos novos, para cada ano, excluindo o não melanoma (BRAY $F$, et al., 2018; INCA, 2017b).

Com progressão do carcinoma epidermóide na região glútea, tem-se como proposta cirúrgica a hemicorporectomia $(\mathrm{HC})$, que envolve amputação da pelve e extremidades inferiores por desarticulação através da coluna lombar com transecção concomitante da aorta, veia cava inferior e medula espinhal (JANIS JE, 2009). A HC é um procedimento descrito na literatura como raro, associado a efeitos devastadores, incluindo imagem corporal alterada, distúrbio da função autonômica e várias consequências nas habilidades funcionais físicas, sendo utilizada para o tratamento de patologias oncológicas do assoalho pélvico; existindo apenas dois relatos de casos em trauma (KREDEL FE, 1951; WARR SP, et al., 2018).

A cirurgia de HC é realizada para aliviar o sofrimento e melhorar tanto a qualidade quanto a quantidade da vida do paciente quando todos os outros cursos de tratamento foram esgotados. Candidatos a hemicorporectomia são pacientes que sofrem; que experimentaram muitas hospitalizações e vivem com dor crônica (MASTERSON EL, 2006). Em suma, uma cirurgia de hemicorporectomia bem-sucedida pode ser vista como um procedimento que melhora a qualidade de vida do paciente a longo prazo, contudo, é eticamente insustentável em casos que não ocorra risco de vida ou que ajam alternativas terapêuticas, por conta da extrema modificação corporal (JANKOWSKI J e CAMPO-ENGELSTEIN L, 2014).

Neste sentido, a enfermagem atual desenha-se como ciência e vem buscando construir seus valores e estabelecer suas teorias por intermédio da implementação da Sistematização da Assistência de Enfermagem (SAE), objetivando romper com os mitos e empirismos envoltos ao processo de cuidar sem embasamento científico (ANDRADE JS, 2016), isto é, a SAE, por meio da utilização do Processo de Enfermagem (PE) e de outros métodos de organização do cuidado, vem adquirindo espaço diante as discussões dos pensadores e pesquisadores de Enfermagem como importante instrumento de consolidação da enfermagem como ciência (MOSER DC, et al., 2018).

Nesse contexto, o PE requer do enfermeiro interesse em conhecer o paciente como indivíduo, utilizando para isto seus conhecimentos e habilidades, em especial no atendimento ao paciente com câncer, que carece do enfermeiro um cuidado complexo em função de características peculiares a este adoecimento (SOUSA PHG, et al., 2018). Diante do exposto este trabalho tem como objetivo apresentar um descrever a assistência de enfermagem um paciente com proposta cirúrgica de hemicorporectomia.

\section{DETALHAMENTO DO CASO}

Trata-se de um estudo qualitativo, retrospectivo, do tipo relato de caso, uma vez que este tipo de estudo favorece uma visão holística sobre os acontecimentos da vida real (YIN RK, 2015). Foi realizado em julho de 2019, no Departamento de Arquivo Médico e Estatística (DAME) de um hospital de referência no tratamento do 
câncer no estado do Pará. Utilizou-se para a coleta de dados um roteiro estruturado composto por: identificação do paciente, antecedentes pessoais e familiares, hábitos de vida, condições socioeconômicas e culturais, exame físico e plano de cuidados do paciente de acordo com a North American Nursing Diagnosis Association (NANDA-I), Nursing Interventions Classification (NIC) e Nursing Outcomes Classifiction (NOC). A análise dos dados será subsidiada pelo Processo de Enfermagem e demais bases conceituais que debatem a temática. Neste relato de caso foram respeitados os preceitos éticos contidos na Resolução no 466/12 e 510/16. Para

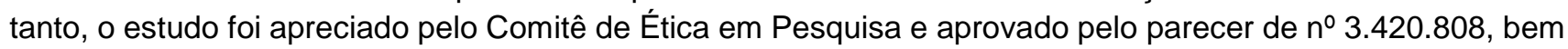
como apresentado o Termo de Compromisso de Utilização de Dados (TCUD).

O relato do caso será apresentado a seguir em dois tópicos: descrição do caso clínico e planejamento da assistência de enfermagem. O paciente é representado pelas iniciais S. J. B. M., possui 31 anos, masculino, solteiro, estudante, natural e residente no município de Vigia (PA). O cliente deu entrada no Hospital, para internação na clínica oncoabdomen no dia 19/03/2018, tendo como diagnóstico carcinoma espinocelular e proposta cirúrgica de hemicorporectomia.

No momento da internação chegou em cadeira de rodas, encontrava-se consciente e orientado em tempo e pessoa, contactuante, com padrão de fala preservado, respirando em ar ambiente, apresentava-se calmo. $O$ paciente referiu não ter realizado cirurgias anteriores, dialítico, com espinha bífida desde a infância, locomovese em cadeira de rodas. Nos últimos meses desenvolveu bexiga neurogênica, evoluindo com lesão por pressão sacral e trocantérica esquerda, apresentando degeneração malina em virtude do carcinoma em nádega esquerda, queixando dor local leve a moderada, sangramento e do odor. Em relação a antecedentes mórbidos familiares, não relatou doenças crônicas; aos antessentes pessoas não informou ter tido doenças da quadra infantil ou possuir demais comorbidades. Negou alergia a medicação ou alimentação até o momento, bem como etilismo ou tabagismo. Relatou perda de peso, mas mantem dieta por via oral, realizando pelo menos 3 refeições ao dia, ingerindo parcialmente ou totalmente alimento com ingesta hídrica de pelo menos 8 copos de $200 \mathrm{ml}$ por dia. Relata sono alterado, relata insônia leve que desregula o período de dormir. Eliminação vesical presente, em fralda e evacuação espontânea sólida.

Ao exame físico, apresentava-se com estado geral regular, acianótico, anictérico, afebril, hipocorado. Apresentava couro cabeludo integro, seborreico, cavidade oral integral, ausência de mucosite e dentição integras. Apresentava tórax normal, simétrico, com boa expansibilidade, eupnéico. À ausculta cardíaca com bulhas normofonéticas em dois tempos, não detectada presença de sopro sistólico, normocárdico, hipotenso; à ausculta pulmonar apresentavam murmúrios vesiculares presentes bilateralmente e ausência de ruídos adventícios. Na inspeção do abdome, plano, sem alteração, na ausculta observou-se presença de ruídos hidroaéreos hiperativos, palpação se apresentava flácido e indolor a palpação superficial, e timpânico a percussão. A região dorsal sacral e glúteo com ferida ulcerativa malina extensa maior que $15 \mathrm{~cm}$, com pele perilesão hiperemiada, descamativa, friável, com presença de necrose de liquefação e exsudato em moderada quantidade, envolvimento tecido subcutâneo, com profundidade superficial a moderada, com epíbole e formação irregular, com odor de grau II. Os membros inferiores apresentavam importante hipotrofia, limitação de movimento de extensão e presença de lesão por pressão grau 1.

Dentre os primeiros exames solicitados, o eletrocardiograma resultou em ritmo sinusal e regular, sem alterações. As principais alterações encontradas no hemograma foram baixos índices em células vermelhas e componentes, apontado anemia, bem como elevada taxa de ureia sugerindo algum tipo de comprometimento renal e creatinina, enzina que é responsável por catabolizar o metabolismo de células musculares. Além disso indicou leucocitose que pode ser explicado pela progressão da doença oncológica e plaquetopenia o que justifica o sangramento da ferida oncológica.

\section{Planejamento da assistência de enfermagem}

Apesar do principal motivo da internação do paciente tenha sido em decorrência do agravamento do carcinoma, o foco da equipe de enfermagem deve dar-se de maneira integral, isto é, tanto em relação a prestação da assistência ao indivíduo pautado na patologia diagnosticada pelo médico, quando nas demais necessidade humanas adentrando no campo das interações, reflexões e conhecimento no contexto social, cultural e espiritual. 
O planejamento da assistência de enfermagem deu-se de acordo com os principais diagnósticos identificados junto ao paciente, subsidiado pelas ligações NANDA-NOC-NIC para respaldar a prática da equipe de enfermagem. Os diagnósticos e intervenções para um determinado resultado esperado estão dispostos a seguir:

1. Diagnóstico: Disposição para conhecimento de saúde melhorado, caracterizado por desejo de melhorar a aprendizagem. NOC: Promoção da saúde. NIC: Ensino: individuo; Ensino: atividades/exercícios; Ensino: Dieta; Ensino: Procedimento. 2. Diagnóstico: Risco de distúrbio na identidade pessoal, com fator de risco a alteração no papel social; baixa autoestima; discriminação. NOC: Identidade. NIC: Melhora da autopercepção; Melhora do enfrentamento; Esclarecimento de valores. 3. Diagnóstico: Risco de baixa autoestima situacional, com fator de risco a alteração da imagem corporal e diminuição do controle sobre o ambiente. NOC: Adaptação à Deficiência Física; Ajuste Psicossocial: Mudança de Vida; Autoestima; Resiliência Pessoal. NIC: Monitorar presença de sinais e sintomas de abuso emocional; reforçar os pontos positivos pessoais identificados pelo paciente; proporcionar experiências que aumentem a autonomia do paciente, conforme apropriado; transmitir confiança na capacidade do paciente para lidar com a situação; recompensar ou elogiar o progresso do paciente na direção das metas; monitorar a frequência de verbalizações autonegativas. 4. Diagnóstico: Padrão de sexualidade ineficaz, relacionado a processo patológico, caracterizado por alteração e dificuldade com atividade sexual. NOC: Identidade sexual. NIC: Ensino: individuo; Redução da ansiedade; Melhora do enfrentamento; Aconselhamento; Melhora da autopercepção; Esclarecimento de valores. 5. Diagnóstico: Risco de infecção, com fator risco a alteração na integridade da pele, associado à doença crônica. NOC: Detecção de riscos; Controle de riscos. NIC: Monitoração nutricional; Cuidados com lesões; Supervisão da pele; Cuidados com sondas; Irrigação de lesões; Promoção da saúde oral.

Este plano pautou-se principalmente no caráter psicossocial do paciente, preparando-o para a cirurgia, sem esquecer o fator biológico. Durante a avaliação analisou-se continuamente a efetividade das intervenções realizadas tendo em vista o processo de modificação futura a qual o paciente submeter-se-ia no intuito de prepará-lo para sua nova imagem. Durante o processo de reavaliação da assistência apresentou melhora do entendimento sobre seu contexto e enfretamento.

\section{DISCUSSÃO}

A utilização do PE viabilizou o cuidado dinâmico e humanizado, proporcionando segurança ao paciente e equipe de enfermagem. Com ela pôde-se levar em consideração fatores que permitem a reflexão sobre valores e atitudes o que permite um pensar e agir diferenciado, entendendo que o paciente é ser com crenças que não podem ser ignorados durante seu adoecimento (ARRIEIRA ICO, et al., 2017). Esta visão de intervenção integral da enfermagem pode melhorar notavelmente a qualidade de vida dos pacientes, diminuir a incidência de complicações e aumentar a sua sobrevida, o que é merecedor de popularização e aplicação clínica (GOU Y, et al., 2019).

No processo perioperatório, os enfermeiros tendem a direcionar os pacientes não somente na compreensão do tratamento e enfretamento de possíveis mudanças, mas emocionalmente, mediante ao suporte na expressão moral dos seus valores, realizando assim a consolidação do vínculo de confiança frente ao amparo as dificuldades inerentes ao momento (FRUET IMA, et al., 2017). Além destas condutas poderem contribuir com a melhorar a qualidade de vida das pessoas com câncer (Silva MR et al., 2017).

O raciocínio critico unido ao processo de enfermagem pautado na cientificidade, no raciocínio clínico e na prestação da assistência humanizada subsidiam o melhor planejamento da assistência do paciente com vistas as necessidades humanas prioritárias afetadas e identificação das respostas do indivíduo a intervenção com vistas a recuperação da saúde ou a melhoria do bem-estar (Silva MR et al., 2017). Posto isto, é importante enfatizar o cuidado emocional na dor oncológica, representando uma síndrome que vai além da nocicepção (lesão), pois envolvem também fatores emocionais, sociais e espirituais (ARRIEIRA ICO, et al., 2017).

Logo a SAE, mostra-se como método de organizar todos esses cuidados e saberes, deste modo, vem ganhando espaço nas discussões dos pesquisadores de Enfermagem como importante instrumento de consolidação da Enfermagem como ciência (MOSER DC, et al., 2018). 
Este pensar diferenciado da enfermagem se faz fundamental ao se abordar o carcinoma visto que se constitui como verdadeiro desafio em virtude do seu alto poder de invasão locorregional e complicações locais, logo a hemicorporectomia visa o princípio curativo e melhora da qualidade de vida, onde os aspectos psicológicos precisam ser cuidados (Menezes TT et al., 2018).

\section{CONSIDERAÇÕES FINAIS}

A Sistematização da Assistência de Enfermagem possibilitou realizar um planejamento mais individualizado com melhor acolhimento e humanização. Como contribuição do estudo destacamos a utilização da taxonomia de ligações NANDA-NOC-NIC como ferramenta para direcionamento das intervenções de enfermagem considerando os fatores sociais, culturais e espirituais, obtendo-se efeitos eficazes, com melhora significativa no enfrentamento da doença e continuidade no tratamento. Como limitações e dificuldades da pesquisa apontase os poucos estudos acerca desta cirurgia para comparação e reflexão sobre os cuidados implementados pela enfermagem, evidenciando seu ineditismo dado a raridade do procedimento. Por fim, enfatiza-se a necessariedade da realização de estudos pautados da SAE como forma de partilhar formas de cuidados e repensar as práticas em saúde.

\section{REFERÊNCIAS}

1. ANDRADE JS. Sistematização da Assistência de Enfermagem em uma unidade de urgência e emergência: autonomia e visibilidade da equipe de enfermagem. Rev Bras Enferm, 2016; 58(3): 261-5.

2. ARRIEIRA ICO, et al. O sentido do cuidado espiritual na integralidade da atenção em cuidados paliativos. Rev Gaúcha Enferm, 2017; 38(3): e58737.

3. BRAY F, et al. Global Cancer Statistics 2018: GLOBOCAN Estimates of Incidence and Mortality Worldwide for 36 Cancers in 185 Countries. Cancer J Clin, 2018; 68: 394-424.

4. FRUET IMA, et al. Aplicabilidade da Moral Distress Scale adaptada no cenário da enfermagem em hemato-oncologia. Rev Gaúcha Enferm, 2017; 38(4): e63060.

5. GOU Y, et al. Analysis on Effects of Comprehensive Nursing Care Applied in Interventional Therapy for Patients with Liver Cirrhosis and Liver Cancer. Iran J Public Health, 2019; 48(3): 494-500.

6. INSTITUTO NACIONAL DE CÂNCER (INCA). Estimativa 2018: incidência de câncer no Brasil. Rio de Janeiro, 2017b; $128 \mathrm{p}$.

7. JANIS JE. A 25-year experience with hemicorporectomy for terminal pelvic osteomyelitis. Plast Reconstr Surg, 2009; 124(4): 1165-76.

8. JANKOWSKI J, CAMPO-ENGELSTEIN L. A Better Half: The Ethics of Hemicorporectomy Surgery. Journal of Bioethical Inquirys, 2014; 11(3).

9. KREDEL FE. The role of pelvic evisceration in surgery (Discussion). Surgery, 1951; 30: 76-94.

10. MASTERSON EL. Hemicorporectomy: A humane option or a step too far? Irish. Journal of Orthopaedic Surgery and Trauma, 2006; 2: 31-3.

11. MENEZES TT et al. Fechamento de hemicorporectomia com utilização do retalho subtotal unilateral da coxa: relato de caso. Rev bras cir plást, 2018; 33(1):130-134.

12. MOSER DC, et al. Sistematização da Assistência de Enfermagem: percepção dos enfermeiros. Rev Fun Care Online, 2018; 10(4): 998-1007.

13. SILVA MR, et al. Mapeamento dos diagnósticos, resultados e intervenções de enfermagem de uma unidade oncológica. Rev enferm UERJ, 2017; 25:e15133.

14. SOUSA PHG, et al. Diagnósticos de enfermagem em unidade de Terapia intensiva: revisão integrativa. Cultura de los Cuidados, 2018; 22(52): 223-31.

15. WARR SP, et al. European Journal of Orthopaedic Surgery \& Traumatology, 2018; 28: 735-739.

16. YIN RK. Estudo de caso: planejamento e métodos. Tradução Cristhian Matheus Herrera. 5 ed. Porto Alegre: Bookman, $2015 ; 290 \mathrm{p}$. 